\title{
THE LIGHTCONE GAUSS MAP AND THE LIGHTCONE DEVELOPABLE OF A SPACELIKE CURVE IN MINKOWSKI 3-SPACE
}

\author{
SHYUICHI IZUMIYA, DONGHE PEI* and TAKASI SANO \\ Department of Mathematics, Faculty of Science, Hokkaido University, Sapporo 060, Japan
}

(Received 18 May, 1998)

\begin{abstract}
We define the notion of lightcone Gauss maps, lightcone pedal curves and lightcone developables of spacelike curves in Minkowski 3-space and establish the relationships between singularities of these objects and geometric invariants of curves under the action of the Lorentz group.

1991 Mathematics Subject Classification. 53B30, 58C28, 57R70
\end{abstract}

1. Introduction. There are several articles concerning 'generic differential geometry' in Euclidean space $[\mathbf{1 - 5}, \mathbf{8}, \mathbf{1 0}$, etc.]. The main tools in these articles are the distance-squared functions and the height functions on submanifolds. In this paper we introduce the notion of lightcone height functions and Lorentzian distancesquared functions on spacelike curves in Minkowski 3-space. We also define the notion of lightcone Gauss maps, lightcone pedal curves and lightcone developables of spacelike curves and establish the relationships between singularities of these subjects and geometric invariants of curves under the action of the Lorentz group as applications of standard techniques of singularity theory for the above functions.

On the other hand, there exists a special subject in Minkowski 3-space, called a lightlike surface, which is defined to be a surface tangent to the light cone at any point. We remark that any lightlike surface is the lightcone developable of a spacelike curve. Thus the main theorem (Theorem B) gives a generic classification of singularities of lightlike surfaces. For the basic notions in Lorentzian geometry, see [9].

Let $\mathbb{R}^{3}=\left\{\left(x_{1}, x_{2}, x_{3}\right) \mid x_{1}, x_{2}, x_{3} \in \mathbb{R}\right\}$ be a 3-dimensional vector space, $\boldsymbol{x}=\left(x_{1}, x_{2}, x_{3}\right)$ and $\boldsymbol{y}=\left(y_{1}, y_{2}, y_{3}\right)$ two vectors in $\mathbb{R}^{3}$, the pseudo scalar product of $\boldsymbol{x}$ and $\boldsymbol{y}$ is defined by $\langle\boldsymbol{x}, \boldsymbol{y}\rangle=-x_{1} y_{1}+x_{2} y_{2}+x_{3} y_{3}$. We call $\left(\mathbb{R}^{3},\langle\rangle,\right)$ a 3 -dimensional pseudo Euclidean space, or Minkowski 3-space. We write $\mathbb{R}_{1}^{3}$ instead of $\left(\mathbb{R}^{3},\langle\rangle,\right)$.

We say that a vector $\boldsymbol{x}$ in $\mathbb{R}_{1}^{3}$ is spacelike, lightlike or timelike if $\langle\boldsymbol{x}, \boldsymbol{x}\rangle>0$, $\langle\boldsymbol{x}, \boldsymbol{x}\rangle=0$ or $\langle\boldsymbol{x}, \boldsymbol{x}\rangle<0$ respectively.

Let $\gamma: I \longrightarrow \mathbb{R}_{1}^{3} ; \gamma(t)=\left(x_{1}(t), x_{2}(t), x_{3}(t)\right)$ be a smooth regular curve in $\mathbb{R}_{1}^{3}$ (i.e., $\dot{\gamma}(t) \neq 0$ for any $t \in I)$, where $I$ is an open interval. The curve $\gamma$ is called a spacelike curve if $\langle\dot{\gamma}(t), \dot{\gamma}(t)\rangle>0$, for any $t \in I$. The norm of the vector $\boldsymbol{x} \in \mathbb{R}_{1}^{3}$ is defined by $\|\boldsymbol{x}\|=\sqrt{|\langle\boldsymbol{x}, \boldsymbol{x}\rangle|}$. The arc-length of a spacelike curve $\gamma$, measured from $\gamma\left(t_{0}\right), t_{0} \in I$ is

$$
s(t)=\int_{t_{0}}^{t}\|\dot{\gamma}(t)\| d t .
$$

*On leave from Department of Mathematics, North East Normal University, Chang Chun 130024, P.R. China. 
Then a parameter $s$ is determined such that $\left\|\gamma^{\prime}(s)\right\|=1$, where $\gamma^{\prime}(s)=d \gamma / d s(s)$. Consequently we say that a spacelike curve $\gamma$ is parameterized by arc-length if it satisfies $\left\|\gamma^{\prime}(s)\right\|=1$. Throughout the remainder of this paper we assume the parameter $s$ of $\gamma$ is the arc-length parameter. Let us denote $\boldsymbol{t}(s)=\gamma^{\prime}(s)$, and we call $\boldsymbol{t}(s)$ a unit tangent vector of $\gamma$ at $s$. We define the curvature by $k(s)=\sqrt{\left|\left\langle\gamma^{\prime \prime}(s), \gamma^{\prime \prime}(s)\right\rangle\right|}$. If $k(s) \neq 0$ then the unit principal normal vector $\mathbf{n}$ of the curve $\gamma$ at $s$ is given by $\gamma^{\prime \prime}(s)=k(s) \cdot \boldsymbol{n}(s)$. The signature of $\boldsymbol{x}$ is defined to be

$$
\operatorname{sign}(\boldsymbol{x})= \begin{cases}1 & \boldsymbol{x} \text { is spacelike } \\ 0 & \boldsymbol{x} \text { is lightlike } \\ -1 & \boldsymbol{x} \text { is timelike }\end{cases}
$$

We write $\delta(\gamma(s))=\operatorname{sign}(\boldsymbol{n}(s))$.

For any $\boldsymbol{x}=\left(x_{1}, x_{2}, x_{3}\right), \boldsymbol{y}=\left(y_{1}, y_{2}, y_{3}\right) \in \mathbb{R}_{1}^{3}$, the pseudo vector product of $\boldsymbol{x}$ and $\boldsymbol{y}$ is defined as follows:

$$
\boldsymbol{x} \wedge \boldsymbol{y}=\left|\begin{array}{ccc}
-e_{1} & e_{2} & e_{3} \\
x_{1} & x_{2} & x_{3} \\
y_{1} & y_{2} & y_{3}
\end{array}\right|=\left(-\left(x_{2} y_{3}-x_{3} y_{2}\right), x_{3} y_{1}-x_{1} y_{3}, x_{1} y_{2}-x_{2} y_{1}\right)
$$

The unit vector $\boldsymbol{b}(s)=\boldsymbol{t}(s) \wedge \boldsymbol{n}(s)$ is called a unit binormal vector of the curve $\gamma$ at $s$. Since $\boldsymbol{t}(s)$ is spacelike, we have $\langle\boldsymbol{b}(s), \boldsymbol{b}(s)\rangle=-\delta(\gamma(s))$ and $\operatorname{sign}\left(\gamma^{\prime}(s)\right)=1$. Then the following Frenet-Serret type formula holds:

$$
\left\{\begin{array}{l}
\boldsymbol{t}^{\prime}(s)=k(s) \cdot \boldsymbol{n}(s) \\
\boldsymbol{n}^{\prime}(s)=-\delta(\gamma(s)) \cdot k(s) \cdot \boldsymbol{t}(s)+\tau(s) \cdot \boldsymbol{b}(s) \\
\boldsymbol{b}^{\prime}(s)=\tau(s) \cdot \boldsymbol{n}(s)
\end{array}\right.
$$

where $\tau(s)$ is the torsion of the curve $\gamma$ at $s$ (cf. [6]). This is the fundamental formula for the study of spacelike curves in $\mathbb{R}_{1}^{3}$; it is, however, useless at the point $\gamma(s)$ with $k(s)=0$. We now denote $N(s)=\gamma^{\prime \prime}(s)$ and $B(s)=\boldsymbol{t}(s) \wedge N(s)$. We simply call $N(s)$ a principal normal vector and $B(s)$ a binormal vector. If $k(s) \neq 0$, then we have $N(s)=k(s) \boldsymbol{n}(s)$ and $B(s)=k(s) \boldsymbol{b}(s)$. It follows that

$$
\langle N(s) \pm B(s), N(s) \pm B(s)\rangle=k^{2}(s)(\delta(\gamma(s))-\delta(\gamma(s)))=0 .
$$

If $k(s)=0$, then $N(s)$ is a lightlike vector, so that any pseudo perpendicular vector in the normal plane of $\gamma(s)$ is parallel to $N(s)$. We can prove that $N(s) \pm B(s) \neq \mathbf{0}$. This means that $N(s) \pm B(s)$ is a lightlike vector which is parallel to the vector $N(s)$ for $s \in I$ with $k(s)=0$. Define

$$
\begin{gathered}
S_{+}^{1}=\left\{x \in \mathbb{R}_{1}^{3} \mid x=\left(1, x_{2}, x_{3}\right), x_{2}^{2}+x_{3}^{2}=1\right\}, \\
C_{p}=\left\{x=\left(x_{1}, x_{2}, x_{3}\right) \in \mathbb{R}_{1}^{3} \mid-\left(x_{1}-p_{1}\right)^{2}+\left(x_{2}-p_{2}\right)^{2}+\left(x_{3}-p_{3}\right)^{2}=0\right\},
\end{gathered}
$$

where $p=\left(p_{1}, p_{2}, p_{3}\right)$. We call $S_{+}^{1}$ a lightlike unit circle and $C_{p}^{*}=C_{p}-\{p\}$ a lightcone at the vertex $p$. For any lightlike vector $\boldsymbol{x}=\left(x_{1}, x_{2}, x_{3}\right)$, we write $\tilde{\boldsymbol{x}}=\left(1, \frac{x_{2}}{x_{1}}, \frac{x_{3}}{x_{1}}\right) \in S_{+}^{1}$. With this notation, we have $N(s) \widetilde{ \pm} B(s)=\widetilde{N(s)}$ if $k(s)=0$. 
We now define a map $L G_{\gamma}^{+}: I \longrightarrow S_{+}^{1}$ by

$$
L G_{\gamma}^{+}(s)=N(s) \widetilde{+} B(s)
$$

and a curve $L P_{\gamma}^{+}: I \longrightarrow C^{*}$ by

$$
L P_{\gamma}^{+}(s)=\langle\gamma(s), N(s) \widetilde{+} B(s)\rangle \cdot(N(s) \widetilde{+} B(s)),
$$

where we may assume that $\gamma(s) \neq 0$. Under the assumption that $k(s) \neq 0$, we also define a ruled surface $L D_{\gamma}^{+}: I \times \mathbb{R} \longrightarrow \mathbb{R}_{1}^{3}$ by

$$
L D_{\gamma}^{+}(s, u)=\gamma(s)+u(\boldsymbol{n}(s)+\boldsymbol{b}(s)) .
$$

We call $L G_{\gamma}^{+}$the lightcone Gauss map and $L P_{\gamma}^{+}$the lightcone pedal curve (or lightcone dual curve) of $\gamma$. We also call $L D_{\gamma}^{+}$the lightcone developable of $\gamma$. The geometric properties of these subjects will be discussed in $\S 3$. We can also define $L G_{\gamma}^{-}, L P_{\gamma}^{-}$and $L D_{\gamma}^{-}$exactly in the same way as the above. Since these have the same properties as those of the above, we do not consider these here.

Let $\gamma: S^{1} \longrightarrow \mathbb{R}_{1}^{3}$ be a spacelike curve with $\gamma^{\prime \prime}(s) \neq 0$. We consider the following properties of $\gamma$.

(A 1) The number of points $p$ of $\gamma\left(S^{1}\right)$ where the lightcone at $p$ has two-point contact with the principal normal curve $\gamma^{\prime \prime}$ is finite.

(A 2) There is no point $p$ of $\gamma\left(S^{1}\right)$ where the lightcone at $p$ has greater than two-point contact with the principal normal curve $\gamma^{\prime \prime}$.

In $\S 3$, under the assumption that $k\left(s_{0}\right) \neq 0$, we shall show that there exist just two lightcones at points

$$
v_{ \pm}=\gamma\left(s_{0}\right)+\frac{1}{k\left(s_{0}\right) \delta\left(\gamma\left(s_{0}\right)\right)}\left(\boldsymbol{n}\left(s_{0}\right) \pm \boldsymbol{b}\left(s_{0}\right)\right)
$$

such that $\gamma$ has three-point contact with the lightcones at $v_{ \pm}$. We call each lightcone an osculating lightcone of $\gamma$.

(A 3) The number of points at which $\gamma$ has four-point contact with the osculating lightcone is finite.

(A 4) There is no osculating lightcone with which $\gamma$ has at least five-point contact at a point.

Our main results are formulated as follows.

TheOREM A. (1) Let $\operatorname{Imm}_{s}\left(S^{1}, \mathbb{R}_{1}^{3}\right)$ be a space of spacelike curves equipped with Whitney $C^{\infty}$-topology. Then the set of curves which satisfy (A 1) and (A 2) is a residual set in $\operatorname{Imm}_{s}\left(S^{1}, \mathbb{R}_{1}^{3}\right)$.

(2) Let $\operatorname{Imm}_{s}^{+}\left(S^{1}, \mathbb{R}_{1}^{3}\right)$ be a space of spacelike curves with $k(s) \neq 0$ equipped with Whitney $C^{\infty}$-topology. Then the set of curves which satisfy (A 3) and (A 4) is a residual set in $\operatorname{Imm}_{s}^{+}\left(S^{1}, \mathbb{R}_{1}^{3}\right)$.

TheOREM B. (1) Under the assumption of (A 1) and (A 2),

(a) the lightcone Gauss map $L G_{\gamma}^{+}$has a fold point at $s_{0}$ if and only if $k\left(s_{0}\right)=0$,

(b) the lightcone pedal curve $L P_{\gamma}^{+}$has a cusp point at $L P_{\gamma}^{+}\left(s_{0}\right)$ if and only if $k\left(s_{0}\right)=0$.

(2) Assume (A 3) and (A 4). 
(a) The lightcone developable $L D_{\gamma}^{+}$is nonsingular along the curve $\gamma(s)$. Moreover, if we consider another lightcone developable $L D_{\gamma}^{-}$, these surfaces intersect transversally along the curve $\gamma(s)$.

(b) The lightcone developable $L D_{\gamma}^{+}$is locally diffeomorphic to the cuspidal edge at $\gamma\left(s_{0}\right)+u_{0}\left(\boldsymbol{n}\left(s_{0}\right)+\boldsymbol{b}\left(s_{0}\right)\right)$ if and only if $u_{0}=\frac{1}{k\left(s_{0}\right) \delta\left(\gamma\left(s_{0}\right)\right)}$. Moreover, the locus of the vertices of the osculating lightcones $\gamma(s)+\frac{1}{k(s) \delta(\gamma(s))}(\boldsymbol{n}(s)+\boldsymbol{b}(s))$ is the cuspidal edge.

(c) The lightcone developable $L D_{\gamma}^{+}$is locally diffeomorphic to the swallow tail at $\left.\gamma\left(s_{0}\right)+u_{0}\left(\boldsymbol{n}\left(s_{0}\right)+\boldsymbol{b}\left(s_{0}\right)\right)\right)$ if and only if $u_{0}=\frac{1}{k\left(s_{0}\right) \delta\left(\gamma\left(s_{0}\right)\right)}$ and $\left(k^{\prime}-\tau \cdot k\right)(s)=0$.

If we consider a curve $\gamma$ on the $\left(x_{2}, x_{3}\right)$-plane, it is always a spacelike curve. In this case the $\left(x_{2}, x_{3}\right)$-plane is a Euclidean plane, so that the curvature $k(s)$ is the ordinary Euclidean curvature and the torsion $\tau(s)$ is constantly equal to zero. Then $\left(k^{\prime}-\tau \cdot k\right)(s)=k^{\prime}(s)$. This means that the swallowtail point of the rightcone developable of $\gamma$ corresponds to the ordinary vertex of $\gamma$. Figure 1 is the picture of the rightcone developable of the ellipse:

$$
\gamma(t)=(0,2 \cos t, \sin t) \quad(0 \leq t \leq 2 \pi) .
$$

We can easily recognize that there exist four swallowtails.

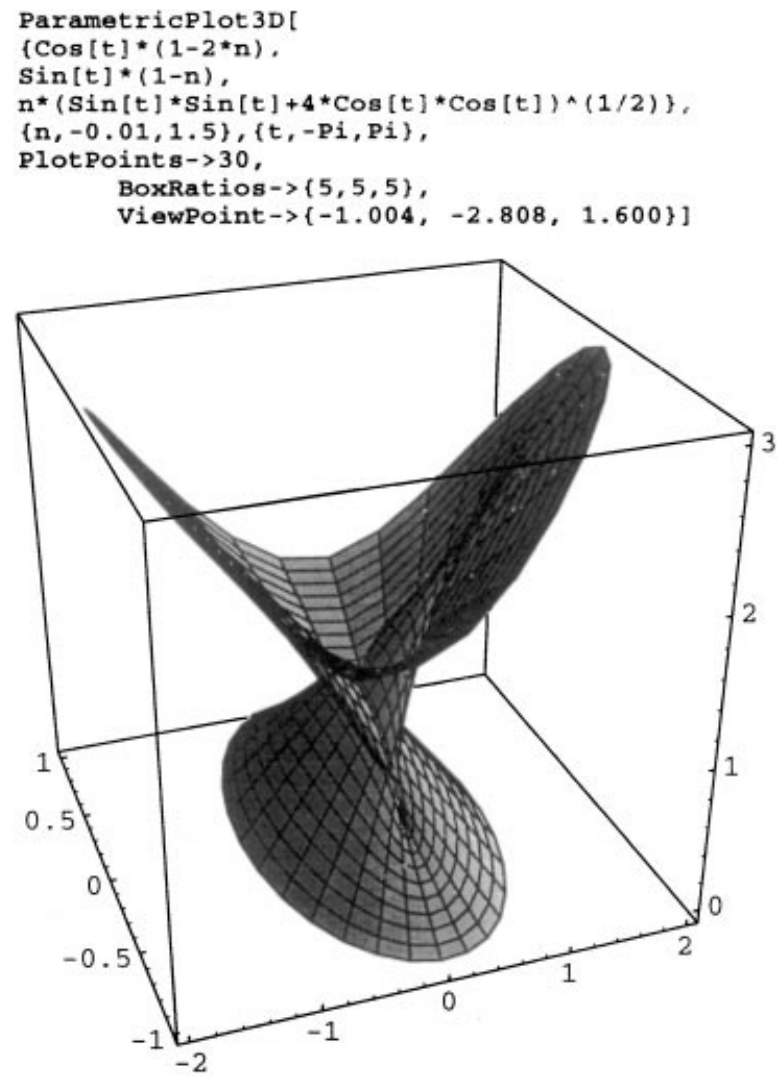

-Graphics3D-

Figure 1 
In [7], M. Kossowski introduced the notion of $S^{1} \times S^{1}$-valued Gauss maps associated with spacelike curves. The authors are much inspired by his paper. Especially, the authors learned the fact that $\boldsymbol{n}(s) \pm \boldsymbol{b}(s)$ is lightlike in his paper. As a matter of fact, the notion of lightcone Gauss maps in this paper is almost the same as that of $S^{1} \times S^{1}$-valued Gauss maps. The basic techniques in this paper depend heavily on those in the attractive book of Bruce and Giblin [4]. In $\$ 2$ we introduce the notion of lightcone height functions and Lorentzian distance-squared functions on spacelike curves and study these properties. The Lorentzian distance-squared function is just a direct analogy of the distance squared function in Euclidean 3 -space. We can consider the Lorentzian focal surfaces and the Lorentzian canal surface of a spacelike curve by using Lorentzian distance squared functions. It is, however, a simple analogy of the Euclidean case. Here, we consider the notion of lightcone developables, which is a special subject in Lorentzian geometry. We study some Lorentzian invariants in $\$ 3$. These invariants are squeezed out by the study of lightcone height functions and Lorentzian distance squared functions in $\S 2$. The proof of Theorem $\mathrm{B}$ is given in $\$ 4$. In $\$ 5$ we consider the generic properties by using the Lorentzian analogy of the notion of Monge-Taylor maps of curves in $[4]$.

2. Lorentzian invariant functions on spacelike curves. In this section we introduce two different families of functions on a spacelike curve which are useful in the study of Lorentzian invariants of spacelike curves.

For a spacelike curve $\gamma: I \longrightarrow \mathbb{R}_{1}^{3}$, we now define a function

$$
H: I \times S_{+}^{1} \longrightarrow \mathbb{R}
$$

by $H(s, v)=\langle\gamma, v\rangle$. We call $H$ a lightcone height function on the spacelike curves $\gamma$. We denote this $h(s)=H_{v_{0}}(s)=H\left(s, v_{0}\right)$, for any fixed $v_{0} \in S_{+}^{1}$. We have the following proposition.

Proposition 2.1. Let $\gamma: I \longrightarrow \mathbb{R}^{3}$ be a unit speed spacelike curve with $\gamma^{\prime \prime}(s) \neq 0$.

(1) $h^{\prime}\left(s_{0}\right)=0$ if and only if $v$ is in the normal plane at $\gamma\left(s_{0}\right)$. Especially, if $k\left(s_{0}\right) \neq 0$, then $v=N\left(s_{0}\right) \pm B\left(s_{0}\right)$.

(2) $h^{\prime}\left(s_{0}\right)=h^{\prime \prime}\left(s_{0}\right)=0$ if and only if $v=N\left(s_{0}\right) \widetilde{ \pm} B\left(s_{0}\right)=\widetilde{N\left(s_{0}\right)}$ and $k\left(s_{0}\right)=0$.

(3) $h^{\prime}\left(s_{0}\right)=h^{\prime \prime}\left(s_{0}\right)=h^{(3)}\left(s_{0}\right)=0$ if and only if $v\left(s_{0}\right)=N(s) \widetilde{ \pm} B\left(s_{0}\right)=\widetilde{N\left(s_{0}\right)}$, and $k\left(s_{0}\right)=\left\langle N^{\prime}\left(s_{0}\right), N\left(s_{0}\right)\right\rangle=0$.

(4) $h^{\prime}\left(s_{0}\right)=h^{\prime \prime}\left(s_{0}\right)=h^{(3)}\left(s_{0}\right)=h^{(4)}\left(s_{0}\right)=0$ if and only if $v=N\left(s_{0}\right) \widetilde{ \pm}\left(s_{0}\right)=$ $\widetilde{N\left(s_{0}\right)}$, and $k\left(s_{0}\right)=\left\langle N^{\prime}\left(s_{0}\right), N\left(s_{0}\right)\right\rangle=\left\langle N^{\prime \prime}\left(s_{0}\right), N\left(s_{0}\right)\right\rangle=0$.

Proof. Let $H: I \times S_{+}^{1} \longrightarrow \mathbb{R}$ be the lightcone height function on the spacelike curve $\gamma: I \longrightarrow \mathbb{R}_{1}^{3}$. Then we have $\frac{\partial H}{\partial s}=\left\langle\gamma^{\prime}(s), v\right\rangle=\langle\boldsymbol{t}(s), v\rangle$, where $v=\left(1, x_{2}, x_{3}\right) \in S_{+}{ }^{1}$. It follows that $\frac{\partial H}{\partial s}=0$ if and only if $\langle v, \boldsymbol{t}\rangle=0$. Especially, if $k(s) \neq 0$, then there exist $\lambda$, $\mu$ such that $v=\lambda(\boldsymbol{n}+\mu \boldsymbol{b})$. Since $\langle v, v\rangle=0$, we have $\delta(\gamma) \cdot \lambda^{2}-\delta(\gamma) \cdot \mu^{2}=0$, so that we have $\lambda^{2}=\mu^{2}$. It follows that we have $v=\tilde{n} \pm \boldsymbol{b}$.

On the other hand, we have $\frac{\partial^{2} H}{\partial s^{2}}=\langle N(s), v\rangle$. If $k(s) \neq 0$, then $\frac{\partial H}{\partial s}=\frac{\partial^{2} H}{\partial s^{2}}=0$ if and only if $v=\tilde{\boldsymbol{n} \pm \boldsymbol{b}}=N(s) \pm B(s)$ and $\langle k \cdot \boldsymbol{n}, v\rangle=0$. This is equivalent to the condition that $v=\widetilde{\boldsymbol{n} \pm \boldsymbol{b}}$ and $k\langle\boldsymbol{n}, \boldsymbol{n \pm \boldsymbol { b }}\rangle=k \delta(\gamma)=0$. Since $\delta(\gamma)= \pm 1$, the above condition 
means that $v=\widetilde{n \pm b}$ and $k=0$. This is a contradiction, so that we have $k(s)=0$; then $N(s)$ is a lightlike vector. Since $\langle v, N(s)\rangle=0, v$ is parallel to $N(s)$. It is equivalent to the fact that $v=\widetilde{N(s)}=N(s) \widetilde{ \pm}(s)$.

Since $\frac{\partial^{3} H}{\partial s^{3}}=\left\langle N^{\prime}(s), v\right\rangle, \frac{\partial H}{\partial s}=\frac{\partial^{2} H}{\partial s^{2}}=\frac{\partial^{3} F}{\partial s^{3}}=0$ if and only if $v=\widetilde{N(s)}=N(s) \widetilde{ \pm}(s)$, $k(s)=0$ and $\left.\left\langle N^{\prime}(s), N(s)\right)\right\rangle \stackrel{\partial s}{=}$.

Moreover, we have $\frac{\partial^{4} H}{\partial s^{4}}=\left\langle N^{\prime \prime}(s), v\right\rangle$. Then $\frac{\partial H}{\partial s}=\frac{\partial^{2} H}{\partial s^{2}}=\frac{\partial^{3} H}{\partial s^{3}}=\frac{\partial^{4} H}{\partial s^{4}}=0$ if and only if $v=N\left(s_{0}\right) \pm B\left(s_{0}\right)=\widetilde{N\left(s_{0}\right)}, k=\left\langle N^{\prime}, N\right\rangle=0$ and $\left\langle N^{\prime \prime}, v\right\rangle=0$. Since $v$ is parallel to $N$, we have $\left\langle N^{\prime \prime}, N\right\rangle=0$.

We now define a function

$$
G: I \times \mathbb{R}_{1}^{3} \longrightarrow \mathbb{R}
$$

by

$$
G(s, v)=\langle\gamma-v, \gamma-v\rangle
$$

We call $G$ the Lorentzian distance-squared function on a spacelike curve $\gamma$. We note that $g(s)=G_{v_{0}}(s)=G\left(s, v_{0}\right)$, for any fixed $v_{0} \in \mathbb{R}_{1}{ }^{3}$. We also have the following proposition.

Proposition 2.2. Let $\gamma: I \longrightarrow \mathbb{R}_{1}^{3}$ be a unit speed spacelike curve with $k(s) \neq 0$.

(1) $g\left(s_{0}\right)=g^{\prime}\left(s_{0}\right)=0$ if and only if $\gamma\left(s_{0}\right)-v\left(s_{0}\right)=\lambda\left(\boldsymbol{n}\left(s_{0}\right) \pm \boldsymbol{b}\left(s_{0}\right)\right)(\lambda \in \mathbb{R}-\{0\})$.

(2) $g\left(s_{0}\right)=g^{\prime}\left(s_{0}\right)=g^{\prime \prime}\left(s_{0}\right)=0$ if and only if $v=\gamma\left(s_{0}\right)+\frac{1}{k\left(s_{0}\right) \cdot \delta\left(\gamma\left(s_{0}\right)\right)}\left(\boldsymbol{n}\left(s_{0}\right) \pm \boldsymbol{b}\left(s_{0}\right)\right)$ and $k\left(s_{0}\right) \neq 0$.

(3) $g\left(s_{0}\right)=g^{\prime}\left(s_{0}\right)=g^{\prime \prime}\left(s_{0}\right)=g^{(3)}\left(s_{0}\right)=0$ if and only if $v=\gamma\left(s_{0}\right)+\frac{1}{k\left(s_{0}\right) \cdot \delta\left(\gamma\left(s_{0}\right)\right)}$ $\left(\boldsymbol{n}\left(s_{0}\right) \pm \boldsymbol{b}\left(s_{0}\right)\right), k\left(s_{0}\right) \neq 0$ and $\left(k^{\prime} \mp k \cdot \tau\right)\left(s_{0}\right)=0$.

(4) $g\left(s_{0}\right)=g^{\prime}\left(s_{0}\right)=g^{\prime \prime}\left(s_{0}\right)=g^{(3)}\left(s_{0}\right)=g^{(4)}\left(s_{0}\right)=0$ if and only if $v=\gamma\left(s_{0}\right)+$ $\frac{\left(\boldsymbol{n}\left(s_{0}\right) \pm \boldsymbol{b}\left(s_{0}\right)\right)}{k\left(s_{0}\right) \cdot \delta\left(\gamma\left(s_{0}\right)\right)}, \quad k\left(s_{0}\right) \neq 0$ and $\left(k^{\prime} \mp k \cdot \tau\right)\left(s_{0}\right)=\left(k^{\prime} \mp k \cdot \tau\right)^{\prime}\left(s_{0}\right)=0$.

Proof. Let $G: I \times \mathbb{R}_{1}^{3} \longrightarrow \mathbb{R}$ be a Lorentzian distance-squared function on the spacelike curve $\gamma: I \longrightarrow \mathbb{R}_{1}^{3}$. Then we have $\frac{\partial G}{\partial s}=2\left\langle\gamma^{\prime}, \gamma-v\right\rangle$. It follows that $\frac{\partial G}{\partial s}=0$ if and only if $\gamma-v=\lambda \cdot \boldsymbol{n}+\mu \cdot \boldsymbol{b}$. Thus $G(s, v)=\frac{\partial G}{\partial s}=0$ if and only if $\gamma-v=\lambda \cdot \boldsymbol{n}+\mu \cdot \boldsymbol{b}$ and $\langle\lambda \cdot \boldsymbol{n}+\mu \cdot \boldsymbol{b}, \lambda \cdot \boldsymbol{n}+\mu \cdot \boldsymbol{b}\rangle=0$. This is equivalent to the condition that $\gamma-v=\lambda(\boldsymbol{n} \pm \boldsymbol{b})$.

On the other hand, since $\frac{\partial^{2} G}{\partial s^{2}}=2\left\langle\boldsymbol{t}^{\prime}, \gamma-v\right\rangle+2\langle\boldsymbol{t}, \boldsymbol{t}\rangle=2\{\langle k \cdot \boldsymbol{n}, \gamma-v\rangle+1\}$, $G=\frac{\partial G}{\partial s}=\frac{\partial^{2} G}{\partial s^{2}}=0$ if and only if $\gamma-v=\lambda(\boldsymbol{n} \pm \boldsymbol{b})$ and $\langle k \cdot \boldsymbol{n}, \lambda(\boldsymbol{n} \pm \boldsymbol{b})\rangle=-1$. The last equality is equivalent to the relation that $k \cdot \lambda \cdot \delta(\gamma)=k \cdot \lambda\langle\boldsymbol{n}, \boldsymbol{n}\rangle=-1$. This means that $v=\gamma+\frac{1}{k \cdot \delta(\gamma)}(\boldsymbol{n} \pm \boldsymbol{b})$ and $k \neq 0$.

Since $\frac{\partial^{3} G}{\partial s^{3}}=2\left\langle(k \cdot \boldsymbol{n})^{\prime}, \gamma-v\right\rangle, G=\frac{\partial G}{\partial s}=\frac{\partial^{2} G}{\partial s^{2}}=\frac{\partial^{3} G}{\partial s^{3}}=0$ if and only if $k \neq 0$ and $\left\langle k^{\prime} \cdot \boldsymbol{n}+k \cdot \boldsymbol{n}^{\prime},-\frac{1}{k \cdot \delta(\gamma)}(\boldsymbol{n} \pm \boldsymbol{b})\right\rangle=0$, which is equivalent to the condition that $k \neq 0$ and $k^{\prime} \cdot \delta(\gamma) \pm k \cdot \tau\langle\boldsymbol{b}, \boldsymbol{b}\rangle=0$. This can be reduced to the condition that $k \neq 0$ and $k^{\prime}- \pm \cdot k \cdot \tau=0$.

Finally, we have $\frac{\partial^{4} G}{\partial s^{4}}=2\left\langle(k \cdot \boldsymbol{n})^{\prime \prime}, \gamma-v\right\rangle+2\left\langle(k \cdot \boldsymbol{n})^{\prime}, \boldsymbol{t}\right\rangle$. Then $\frac{\partial^{4} G}{\partial s^{4}}=0$ if and only if

$$
\begin{gathered}
\left\langle k^{\prime \prime} \cdot \boldsymbol{n}+k^{\prime} \cdot \boldsymbol{n}^{\prime}+\tau \boldsymbol{b}^{\prime} k+\tau \boldsymbol{b} k^{\prime}-2 \delta k k^{\prime} \boldsymbol{t}-\delta k^{2} \boldsymbol{t}^{\prime}+k \tau^{\prime} \boldsymbol{b}, \gamma-v\right\rangle \\
+\left\langle k^{\prime} \boldsymbol{n}+\tau \boldsymbol{b} k-\delta k^{2} \boldsymbol{t}, \boldsymbol{t}\right\rangle=0 .
\end{gathered}
$$


This is equivalent to the condition that

$$
\left\langle k^{\prime \prime} \boldsymbol{n}+k^{\prime} \boldsymbol{n}^{\prime}+k \tau \boldsymbol{b}^{\prime}+\tau \boldsymbol{b} k^{\prime}-2 \delta k k^{\prime} \boldsymbol{t}-\delta k^{3} \boldsymbol{n}+k \tau^{\prime} \boldsymbol{b}, \gamma-v\right\rangle-\delta k^{2}=0 .
$$

Therefore $G=\frac{\partial G}{\partial s}=\frac{\partial^{2} G}{\partial s^{2}}=\frac{\partial^{3} G}{\partial s^{3}}=\frac{\partial^{4} G}{\partial s^{4}}=0$ if and only if

$$
\left\langle k^{\prime \prime} \boldsymbol{n}+k^{\prime} \boldsymbol{n}^{\prime}+\tau \boldsymbol{b}^{\prime} k+\tau \boldsymbol{b} k^{\prime}-\delta k^{3} \boldsymbol{n}+k \tau^{\prime} \boldsymbol{b},-\frac{(\boldsymbol{n} \pm \boldsymbol{b})}{k \cdot \delta}\right\rangle-\delta k^{2}=0 .
$$

By the Frenet-Serret type formula, we can translate this condition into the condition that

$$
\left\langle k^{\prime \prime} \boldsymbol{n}+k^{\prime} \tau \boldsymbol{b}+k \tau^{2} \boldsymbol{n}+k^{\prime} \tau \boldsymbol{b}-\delta k^{3} \boldsymbol{n}+k \tau^{\prime} \boldsymbol{b},-\frac{(\boldsymbol{n} \pm \boldsymbol{b})}{k \cdot \delta}\right\rangle-\delta k^{2}=0 .
$$

Hence we have the condition that $k^{\prime \prime} \mp 2 k^{\prime} \tau \mp k \tau+k \tau^{2}=0$. Under the condition that $k \neq 0, k^{\prime}= \pm k \cdot \tau$, the above condition is equivalent to $\left(k^{\prime} \mp k \cdot \tau\right)^{\prime}=0$.

3. Lorentzian invariants of spacelike curves. In this section we study the geometric properties of the lightcone Gauss maps, the lightcone pedal curve and the lightcone developables of spacelike curves. By the propositions in the last section, we can recognize that the functions $k(s)$ and $\left(k^{\prime} \mp \tau k\right)(s)$ have special meanings. Firstly we have the following proposition.

Proposition 3.1. (1) Let $\gamma: I \longrightarrow \mathbb{R}_{1}^{3}$ be a spacelike curve with $\gamma^{\prime \prime}(s) \neq 0$. Then $k(s) \equiv 0$ if and only if $\gamma^{\prime \prime}(s) \in C_{0}^{*}$.

(2) Let $\gamma: I \longrightarrow \mathbb{R}_{1}^{3}$ be a spacelike curve with $k(s) \neq 0$. Then $\left(k^{\prime} \mp \tau k\right)(s) \equiv 0$ if and only if $p_{ \pm}=\gamma(s)+\frac{1}{k(s) \delta(\gamma)}(\boldsymbol{n}(s) \pm \boldsymbol{b}(s))$ are constant vectors. Under this condition $\gamma(s) \in C_{p_{ \pm}}^{*}$.

Proof. By definition, the assertion (1) holds. In order to prove the assertion (2), we put

$$
P_{ \pm}(s)=\gamma(s)+\frac{1}{k(s) \delta(\gamma)}(\boldsymbol{n}(s) \pm \boldsymbol{b}(s))
$$

then we have

$$
P_{ \pm}^{\prime}(s)=\frac{-\left(k^{\prime} \mp \tau k\right)(s)}{k(s) \delta(s)}(\boldsymbol{n}(s) \pm \boldsymbol{b}(s)) .
$$

It follows that $P_{ \pm}^{\prime}(s) \equiv 0$ if and only if $\left(k^{\prime} \mp \tau k\right)(s) \equiv 0$. Since $(\boldsymbol{n}(s) \pm \boldsymbol{b}(s))$ is lightlike, $\gamma(s) \in C_{p_{ \pm}}$, where $p_{ \pm}=P_{ \pm}(s)$.

COROLlary 3.2. (1) Let $\gamma: I \longrightarrow \mathbb{R}_{1}^{3}$ be a spacelike curve with $\gamma^{\prime \prime}(s) \neq 0$. If $k(s) \equiv 0$, then the lightcone Gauss map of $\gamma$ is constant and the lightcone pedal curve of $\gamma$ is a lightlike line. 
(2) Let $\gamma: I \longrightarrow \mathbb{R}_{1}^{3}$ be a spacelike curve with $k(s) \neq 0$. If $\left(k^{\prime}-\tau k\right)(s) \equiv 0$, then the lightcone developable is the lightcone $C_{p_{+}}$with vertices $p_{+}$, where $p_{+}=\gamma(s)+\frac{1}{k(s) \delta(\gamma)}(\boldsymbol{n}(s)+\boldsymbol{b}(s))$ are the constant points.

Proof. (1) Since $N(s)=\gamma^{\prime \prime}(s)$ is lightlike for any $s \in I$, the locus of $\boldsymbol{t}(s)=\gamma^{\prime}(s)$ is a lightlike line on the pseudo sphere $S_{1}^{2}$, where

$$
S_{1}^{2}=\left\{x \in \mathbb{R}_{1}^{3} \mid\langle x, x\rangle=1\right\}
$$

It follows that $\widetilde{N}(s)$ is constant and the locus of $\langle\gamma(s), \widetilde{N(s)\rangle} \widetilde{N(s)}$ is a lightlike line.

(2) By definition, we have

$$
L D_{\gamma}^{+}(s, u)=\gamma(s)+u(\boldsymbol{n}(s)+\boldsymbol{b}(s))=p_{+}+\left(u-\frac{1}{k(s) \delta(\gamma)}\right)(\boldsymbol{n}(s)+\boldsymbol{b}(s))
$$

so that the image of $L D_{\gamma}^{+}(s, u)$ is in the pair of lightcones with vertices $C_{p_{+}}$. Since $\frac{\partial L D_{\gamma}^{+}}{\partial s}(s, u)=(1-u \delta(\gamma) k(s)) \boldsymbol{t}(s)+\tau(s) \boldsymbol{b}(s)$ and $\frac{\partial L D_{\gamma}^{+}}{\partial u}(s, u)=(\boldsymbol{n}(s)+\boldsymbol{b}(s))$, we have

$$
\frac{\partial L D_{\gamma}^{+}}{\partial s}(s, u) \wedge \frac{\partial L D_{\gamma}^{+}}{\partial u}(s, u)=(u \delta(\gamma) k(s)-1)(\boldsymbol{n}(s)+\boldsymbol{b}(s))+\tau(s) \boldsymbol{t}(s) .
$$

It follows that $L D_{\gamma}^{+}(s, u)$ is an immersed surface at $(s, u)$ with $u \neq \frac{1}{k(s) \delta(\gamma)}$. The image of the set $u=\frac{1}{k(s) \delta(\gamma)}$ consists of the vertices $p_{+}$of the lightcones. This completes the proof.

Let $F: \mathbb{R}_{1}^{3} \longrightarrow \mathbb{R}$ be a submersion and $\gamma: I \longrightarrow \mathbb{R}_{1}^{3}$ a spacelike curve. We say that $\gamma$ and $F^{-1}(0)$ have $k$-point contact for $t=t_{0}$ if the function $g(t)=F \circ \gamma(t)$ satisfies $g\left(t_{0}\right)=g^{\prime}\left(t_{0}\right)=\cdots=g^{(k-1)}\left(t_{0}\right)=0, g^{(k)}\left(t_{0}\right) \neq 0$. By Propositions 2.1, 2.2 and 3.1, we have the following proposition.

Proposition 3.3. (1) Let $\gamma: I \longrightarrow \mathbb{R}_{1}^{3}$ be a unit speed spacelike curve with $\gamma^{\prime \prime}(s) \neq 0$. Then $\gamma^{\prime \prime}$ and the lightcone $C_{0}^{*}$ have 2-point contact for $s=s_{0}$ if and only if $k\left(s_{0}\right)=0$ and $\left\langle N^{\prime}\left(s_{0}\right), N\left(s_{0}\right)\right\rangle \neq 0$.

(2) Let $\gamma: I \longrightarrow \mathbb{R}_{1}^{3}$ be a unit speed spacelike curve with $k(s) \neq 0$. Then $\gamma$ and the lightcone $C_{p+}^{*}$ have 4-point contact for $s=s_{0}$ if and only if $\left(k^{\prime}-k \tau\right)\left(s_{0}\right)=0$ and $\left(k^{\prime}-k \tau\right)^{\prime}\left(s_{0}\right) \neq 0$.

4. Unfoldings of functions of one-variable. In this section we use some general results on the singularity theory for families of function germs. Detailed descriptions are found in the book [4]. Let $F:\left(\mathbb{R} \times \mathbb{R}^{r},\left(s_{0}, x_{0}\right)\right) \rightarrow \mathbb{R}$ be a function germ. We call $F$ an $r$-parameter unfolding of $f$, where $f(s)=F_{x_{0}}\left(s, x_{0}\right)$. We say that $f$ has $A_{k}$-singularity at $s_{0}$ if $f^{(p)}\left(s_{0}\right)=0$, for all $1 \leq p \leq k$, and $f^{(k+1)}\left(s_{0}\right) \neq 0$. We also say that $f$ has $A_{\geq k}$-singularity at $s_{0}$ if $f^{(p)}\left(s_{0}\right)=0$, for all $1 \leq p \leq k$. Let $F$ be an unfolding of $f$ and $f(s)$ have $A_{k}$-singularity $(k \geq 1)$ at $s_{0}$. We denote the $(k-1)$-jet of the partial derivative $\frac{\partial F}{\partial x_{i}}$ at $s_{0}$ by $j^{(k-1)}\left(\frac{\partial F}{\partial x_{i}}\left(s, x_{0}\right)\right)\left(s_{0}\right)=\sum_{j=1}^{k-1} \alpha_{j i} s^{j}$ for $i=1, \ldots, r$. Then $F$ is called a (p) versal unfolding if the $(k-1) \times r$ matrix of coefficients $\left(\alpha_{j i}\right)$ has rank $k-1$ $(k-1 \leq r)$. Under the same condition as the above, $F$ is called a versal unfolding if the $k \times r$ matrix of coefficients $\left(\alpha_{0 i}, \alpha_{j i}\right)$ has rank $k(k \leq r)$, where $\alpha_{0 i}=\frac{\partial F}{\partial x_{i}}\left(s_{0}, x_{0}\right)$. 
We now introduce important sets concerning the unfoldings relative to the above notions. The singular set of $F$ is the set

$$
S_{F}=\left\{(s, x) \mid \frac{\partial F}{\partial s}(s, x)=0\right\} .
$$

The bifurcation set $\mathcal{B}_{F}$ of $F$ is the critical value set of the restriction to $S_{F}$ of the canonical projection $\pi: \mathbb{R} \times \mathbb{R}^{r} \longrightarrow \mathbb{R}$ :

$$
\mathcal{B}_{F}=\left\{x \in \mathbb{R}^{r} \mid \text { there exists } s \text { with } \frac{\partial F}{\partial s}=\frac{\partial^{2} F}{\partial s^{2}}=0 \text { at }(s, x)\right\} .
$$

The discriminant set of $F$ is the set

$$
\mathcal{D}_{F}=\left\{x \in \mathbb{R}^{r} \mid \text { there exists } s \text { with } F=\frac{\partial F}{\partial s}=0 \text { at }(s, x)\right\} .
$$

Then we have the following well-known result (cf. [4]).

Theorem 4.1. Let $F:\left(\mathbb{R} \times \mathbb{R}^{r},\left(s_{0}, x_{0}\right)\right) \rightarrow \mathbb{R}$ be an r-parameter unfolding of $f(s)$ which has $A_{k}$ singularity at $s_{0}$.

(1) Suppose that $F$ is a (p) versal unfolding. morphic to $\{0\} \times \mathbb{R}^{r-1}$.

(a) If $k=2$, then $\left(s_{0}, x_{0}\right)$ is the fold point of $\pi \mid S_{F}$ and $\mathcal{B}_{F}$ is locally diffeo-

(b) If $k=3$, then $\mathcal{B}_{F}$ is diffeomorphic to $C \times \mathbb{R}^{r-2}$.

(2) Suppose that $F$ is a versal unfolding.

(a) If $k=1$, then $\mathcal{D}_{F}$ is locally diffeomorphic to $\{0\} \times \mathbb{R}^{r-1}$.

(b) If $k=2$, then $\mathcal{D}_{F}$ is locally diffeomorphic to $C \times \mathbb{R}^{r-2}$.

(c) If $k=3$, then $\mathcal{D}_{F}$ is locally diffeomorphic to $S W \times \mathbb{R}^{r-3}$.

Here, $C=\left\{\left(x_{1}, x_{2}\right) \mid x_{1}{ }^{2}=x_{2}{ }^{3}\right\}$ is the ordinary cusp and

$$
S W=\left\{\left(x_{1}, x_{2}, x_{3}\right) \mid x_{1}=3 u^{4}+u^{2} v, x_{2}=4 u^{3}+2 u v, x_{3}=v\right\}
$$

is the swallow tail. We also say that a point $x_{0} \in \mathbb{R}^{r}$ is a fold point of a map germ $f:\left(\mathbb{R}^{r}, x_{0}\right) \longrightarrow\left(\mathbb{R}^{r}, f\left(x_{0}\right)\right)$ if there exist diffeomorphism germs $\phi:\left(\mathbb{R}^{r}, x_{0}\right) \longrightarrow\left(\mathbb{R}^{r}, 0\right)$ and $\psi:\left(\mathbb{R}^{r}, f\left(x_{0}\right)\right) \longrightarrow\left(\mathbb{R}^{r}, 0\right)$ such that $\psi \circ \phi\left(x_{1},, x_{r}\right)=\left(x_{1}, \ldots, x_{r-1}, x_{r}^{2}\right)$.

For a unit speed spacelike curve $\gamma: I \longrightarrow \mathbb{R}_{1}^{3}$, we now define a function

$$
\widetilde{H}: I \times S_{+}^{1} \times \mathbb{R} \rightarrow \mathbb{R}
$$

by $\widetilde{H}(s, v, u)=H(s, v)-u=\langle\gamma(s), v\rangle-u$, where $H$ is the lightcone height function. Then we have the following fundamental theorem in this paper.

THEOREM 4.2. Let $\gamma: I \longrightarrow \mathbb{R}_{1}^{3}$ be a unit speed spacelike curve with $\gamma^{\prime \prime}(s) \neq 0$ and $H: I \times S_{+}^{1} \longrightarrow \mathbb{R}$ the lightcone height function on $\gamma$.

(1) If $h(s)=H_{v_{0}}(s)$ has $A_{2}$-singularity at $s_{0}$, then $H$ is the (p)versal unfolding of $h$. 
(2) If $h(s)=\widetilde{H}_{\left(v_{0}, u_{0}\right)}(s)$ has $A_{k}$-singularity $(k=1,2)$ at $s_{0}$, then $\tilde{H}$ is the versal unfolding of $h$.

Let $\gamma: I \longrightarrow \mathbb{R}_{1}^{3}$ be a unit speed spacelike curve with $k(s) \neq 0$ and $G: I \times \mathbb{R}_{1}^{3} \longrightarrow \mathbb{R}$ the Lorentzian distance-squared function. We consider the point $\left(s_{0}, v_{0}\right) \in I \times \mathbb{R}_{1}^{3}$ with $G\left(s_{0}, v_{0}\right)=0$ and $v_{0} \neq \gamma\left(s_{0}\right)$.

(3) If $g(s)$ has $A_{k}$-singularity $(k=1,2,3)$ at $s_{0}$, then $G$ is the versal unfolding of $g:=G_{v_{0}}$.

Proof. (1) We have shown that $H_{v_{0}}$ has $A_{2}$-singularity at $s_{0}$ if and only if there exists a non-zero real number $\lambda$ such that $v=\lambda N\left(s_{0}\right)$ and $k\left(s_{0}\right)=0$. We denote by $\gamma(s)=\left(x_{1}(s), x_{2}(s), x_{3}(s)\right), v=(1, \cos \theta, \sin \theta)$. By definition, we have $H(s, \theta)=$ $-x_{1}(s)+x_{2}(s) \cdot \cos \theta+x_{3}(s) \cdot \sin \theta$. It follows that $\frac{\partial H}{\partial \theta}=-x_{2}(s) \cdot \sin \theta+x_{3}(s) \cdot \cos \theta$ and the 1 -jet of $\frac{\partial H}{\partial \theta}$ at $s_{0}$ is given by $s \cdot-x_{2}^{\prime}\left(s_{0}\right) \cdot \sin \theta+s \cdot x_{3}^{\prime}\left(s_{0}\right) \cdot \cos \theta$. So we require the $1 \times 2$ matrix $\left(-x_{2}(s) \cdot \sin \theta+x_{3}(s) \cdot \cos \theta,-x_{2}^{\prime}\left(s_{0}\right) \cdot \sin \theta+x_{3}^{\prime}\left(s_{0}\right) \cdot \cos \theta\right)$ to have rank 1 which it always does since $-x_{2}^{\prime}\left(s_{0}\right) \cdot \sin \theta+x_{3}^{\prime}\left(s_{0}\right) \cdot \cos \theta \neq 0$. In fact, $-x_{2}^{\prime}\left(s_{0}\right) \cdot \sin \theta+x_{3}^{\prime}\left(s_{0}\right) \cdot \cos \theta$ is equal to the first component of $\gamma^{\prime} \wedge v$. Suppose that $-x_{2}^{\prime}\left(s_{0}\right) \cdot \sin \theta+x_{3}^{\prime}\left(s_{0}\right) \cdot \cos \theta=0$. Since $\left\langle\gamma^{\prime} \wedge v, \gamma^{\prime} \wedge v\right\rangle=\left\langle\boldsymbol{t} \wedge \lambda_{1} N, \boldsymbol{t} \wedge \lambda_{1} N\right\rangle=$ $\lambda^{2}\langle B, B\rangle=\lambda^{2}\langle N, N\rangle=0$, we have

$$
\left\langle\left(0, x_{3}^{\prime}-x_{1}^{\prime} \sin \theta, x_{1}^{\prime} \cos \theta-x_{2}^{\prime}\right),\left(0, x_{3}^{\prime}-x_{1}^{\prime} \sin \theta, x_{1}^{\prime} \cos \theta-x_{2}^{\prime}\right)\right\rangle=0 .
$$

This is equivalent to the condition that

$$
x_{3}^{\prime 2}-2 x_{1}^{\prime} x_{3}^{\prime} \sin \theta+x_{1}^{\prime 2} \sin ^{2} \theta+x_{1}^{\prime 2} \cos ^{2} \theta-2 x_{1}^{\prime} x_{2}^{\prime} \cos \theta+x_{2}^{\prime 2}=0 .
$$

It follows that

$$
x_{3}^{\prime 2}+x_{2}^{\prime 2}+x_{1}^{\prime 2}-2 x_{1}^{\prime}\left(x_{2}^{\prime} \sin \theta+x_{3}^{\prime} \cos \theta\right)=0 \text {. }
$$

Since $-x_{1}^{\prime}+x_{2}^{\prime} \sin \theta+x_{3}^{\prime} \cos \theta=\left\langle\gamma^{\prime}, v\right\rangle=0$, we have $-x_{1}^{\prime 2}+x_{2}^{\prime 2}+x_{3}^{\prime 2}=0$. On the other hand, $-x_{1}^{\prime 2}+x_{2}^{\prime 2}+x_{3}^{\prime 2}=\left\langle\gamma^{\prime}, \gamma^{\prime}\right\rangle=1$. This is a contradiction. Hence $H$ is $(p)$ versal.

(2) In this case we have

$$
\widetilde{H}(s, \theta, u)=H(s, \theta)-u=-x_{1}(s)+x_{2}(s) \cdot \cos \theta+x_{3}(s) \cdot \sin \theta-u .
$$

By Proposition 2.1, $h$ has $A_{1}$-singularity at $s_{0}$ if and only if $v$ is in the normal plane at $\gamma\left(s_{0}\right)$ and $A_{2}$-singularity at $s_{0}$ if and only if there exists a non-zero real number $\lambda_{1}$ such that $v=\lambda_{1}\left(s_{0}\right) N\left(s_{0}\right)$ and $k\left(s_{0}\right)=0$.

Since $\quad \tilde{H}(s, \underset{\sim}{\sim}, u)=H(s, v)-u=\langle\gamma(s), v\rangle-u_{\tilde{\sim}}=-x_{1}(s)+x_{2}(s) \cdot \cos \theta+x_{3}(s)$. $\sin \theta-u$. we have $\frac{\partial H}{\partial \theta}=-x_{2} \cdot \sin \theta+x_{3} \cdot \cos \theta$ and $\frac{\partial H}{\partial u}=1$ at $s_{0}$. So the rank of the $1 \times 2$ matrix $\left(-x_{2} \cdot \sin \theta+x_{3} \cdot \cos \theta, 1\right)$ is 1 . By the same reason as the case (1), we have $-x_{2}^{\prime}\left(s_{0}\right) \cdot \sin \theta+x_{3}^{\prime}\left(s_{0}\right) \cdot \cos \theta \neq 0$. It follows that the rank of the $2 \times 2$ matrix

$$
\left(\begin{array}{ll}
-x_{2} \cdot \sin \theta+x_{3} \cdot \cos \theta & 1 \\
-x_{2}^{\prime} \cdot \sin \theta+x_{3}^{\prime} \cdot \cos \theta & 0
\end{array}\right)
$$

is 2. This means that $\widetilde{H}$ is versal when $h(s)$ has the $A_{k}$-singularity $(k=1,2)$ at $s_{0}$. 
(3) In this case we have

$$
G(s, v)=-\left(x_{1}(s)-v_{1}\right)^{2}+\left(x_{2}(s)-v_{2}\right)^{2}+\left(x_{3}(s)-v_{3}\right)^{2},
$$

where $\gamma(s)=\left(x_{1}(s), x_{2}(s), x_{3}(s)\right)$ and $v=\left(v_{1}, v_{2}, v_{3}\right)$.

Thus we have $\frac{\partial G}{\partial v_{1}}(s)=2\left(x_{1}(s)-v_{1}\right)$, and so the 2-jet at $s_{0}$ is $2\left(s x_{1}^{\prime}\left(s_{0}\right)+\frac{1}{2} s^{2} x_{1}^{\prime \prime}\left(s_{0}\right)\right)$. We also have $\frac{\partial G}{\partial v_{i}}=-2\left(x_{i}(s)-v_{i}\right) \quad(i=2,3)$, and so the 2-jet at $s_{0}$ is $-2\left(s x_{i}^{\prime}\left(s_{0}\right)+\frac{1}{2} s^{2} x_{i}^{\prime \prime}\left(s_{0}\right)\right)$. The condition for versality can be checked as follows.

(i) By Proposition 2.2, $g$ has $A_{1}$-singularity at $s_{0}$ if and only if there exists a non-zero real number $\lambda$ such that $v=\gamma\left(s_{0}\right)-\lambda\left(\boldsymbol{n}\left(s_{0}\right) \pm \boldsymbol{b}\left(s_{0}\right)\right)$ and $k(s) \cdot \lambda \cdot \delta \neq-1$. When $g$ has $A_{1}$-singularity at $s_{0}$, we require the $1 \times 3$ matrix $\left(2\left(x_{1}\left(s_{0}\right)-v_{0,1}\right),-2\left(x_{2}\left(s_{0}\right)-v_{0,2}\right),-2\left(x_{3}\left(s_{0}\right)-v_{0,3}\right)\right)$ to have rank 1 , which it always does since $v_{0} \neq \gamma\left(s_{0}\right)$.

(ii) It also follows from Proposition 2.2 that $g$ has the $A_{2}$-singularity at $s_{0}$ if and only if $v=\gamma+\frac{1}{k\left(s_{0}\right) \cdot \delta(\gamma)}\left(\boldsymbol{n}\left(s_{0}\right) \pm \boldsymbol{b}\left(s_{0}\right)\right)$ and $k(s) \neq 0, k^{\prime}(s) \neq \pm k(s) \cdot \tau$. When $g$ has $A_{2}$-singularity at $s$, we require the $2 \times 3$ matrix

$$
\left(\begin{array}{ccc}
2\left(x_{1}\left(s_{0}\right)-v_{1}\right) & -2\left(x_{2}\left(s_{0}\right)-v_{2}\right) & -2\left(x_{3}\left(s_{0}\right)-v_{3}\right) \\
2 x_{1}^{\prime}\left(s_{0}\right) & -2 x_{2}^{\prime}\left(s_{0}\right) & -2 x_{3}^{\prime}\left(s_{0}\right)
\end{array}\right)
$$

to have rank 2, which follows from the proof of the case (iii).

(iii) By Proposition 2.2, $g$ has $A_{3}$-singularity at $s_{0}$ if and only if $v=\gamma+\frac{(\boldsymbol{n} \pm \boldsymbol{b})}{k \cdot \delta}$ ) and $k\left(s_{0}\right) \neq 0,\left(k^{\prime} \mp k \cdot \tau\right)\left(s_{0}\right)=0$ and $\left(k^{\prime} \mp k \cdot \tau\right)^{\prime}\left(s_{0}\right)=0$. When $g$ has $A_{3}$ singularity at $s$, we require the $3 \times 3$ matrix

$$
\left(\begin{array}{ccc}
2\left(x_{1}\left(s_{0}\right)-v_{1}\right) & -2\left(x_{2}\left(s_{0}\right)-v_{2}\right) & -2\left(x_{3}\left(s_{0}\right)-v_{3}\right) \\
2 x_{1}^{\prime}\left(s_{0}\right) & -2 x_{2}^{\prime}\left(s_{0}\right) & -2 x_{3}^{\prime}\left(s_{0}\right) \\
x_{1}^{\prime \prime}\left(s_{0}\right) & -x_{2}^{\prime \prime}\left(s_{0}\right) & -x_{3}^{\prime \prime}\left(s_{0}\right)
\end{array}\right)
$$

to be nonsingular. The determinant of this matrix is $4 \operatorname{det}\left(\left(v-\gamma\left(s_{0}\right)\right) \gamma^{\prime}\left(s_{0}\right) \gamma^{\prime \prime}\left(s_{0}\right)\right)=$ $4\left\langle\left(v-\gamma\left(s_{0}\right)\right) \wedge \boldsymbol{t}\left(s_{0}\right), k\left(s_{0}\right) \cdot \boldsymbol{n}\left(s_{0}\right)\right\rangle=4\left\langle\lambda\left(\boldsymbol{n}\left(s_{0}\right) \pm \boldsymbol{b}\left(s_{0}\right)\right) \wedge \boldsymbol{t}\left(s_{0}\right), k\left(s_{0}\right) \cdot \boldsymbol{n}\left(s_{0}\right)\right\rangle=4\left\langle-\lambda \boldsymbol{b}\left(s_{0}\right)\right.$ $\left.\mp \lambda \boldsymbol{n}\left(s_{0}\right), k\left(s_{0}\right) \boldsymbol{n}\left(s_{0}\right)\right\rangle= \pm 4 \lambda k\left(s_{0}\right) \delta(\gamma) \neq 0$. But this just says $k\left(s_{0}\right) \neq 0$. This completes the proof.

We now give the proof of Theorem $\mathrm{B}$.

Proof of Theorem B. For the proof of the assertion (1) (a), we consider the set $S_{H}$ associated with the lightcone height function $H$ given by $S_{H}=$ $\left\{(s, v) \in I \times S_{+}^{1} \mid h^{\prime}(s)=0\right\}$. By Proposition 2.1, we have $S_{H}=\{(s, v) \mid v=N(s) \widetilde{ \pm} B(s)\}$. We also consider the canonical projection $\pi: I \times S_{+}^{1} \longrightarrow S_{+}^{1}$ and we can identify $\pi \mid S_{H}$ and the lightcone Gauss map $L G_{\gamma}^{ \pm}$. By the assumption and Propositions 2.1 and 3.3, $h$ has $A_{2}$-singularity at $s_{0}$ if and only if $k\left(s_{0}\right)=0$. It follows from Theorem 4.2 that $H$ is the $(p)$ versal unfolding of $h$ at $s_{0}$.

Therefore Lemma 4.1 , (1) (a) asserts that $\pi \mid S_{H}$ has a fold point at $s_{0}$.

In order to prove the assertion (1) (b), we define a map 


$$
\widetilde{H}: I \times S_{+}^{1} \times \mathbb{R} \longrightarrow \mathbb{R}
$$

by

$$
\widetilde{H}(s, v, u)=H(s, v)-u=\langle\gamma(s), v\rangle-u .
$$

The discriminant set of $\tilde{H}$ is

$$
\mathcal{D}_{\widetilde{H}}=\{(v, u) \mid u=\langle\gamma, v\rangle, v=N(s) \widetilde{\Psi} B(s)\} .
$$

Applying a Lorentzian motion to the curve $\gamma$, we may assume that $\langle\gamma(s), \gamma(s)\rangle \neq 0$. This means that $u=\langle\gamma(s), N(s) \widetilde{ \pm} B(s)\rangle \neq 0$.

We now define a map $\Phi: S_{+}^{1} \times \mathbb{R}_{+} \longrightarrow C_{+}$by $\Phi\left(\left(1, x_{2}, x_{3}\right), \mu\right)=\left(\mu, \mu \cdot x_{2}, \mu \cdot x_{3}\right)$, where $\mathbb{R}_{+}=\{x \in \mathbb{R} \mid x>0\}$ and $C_{+}=\left\{\left(x_{1}, x_{2}, x_{3}\right) \mid x_{1}{ }^{2}=x_{2}{ }^{2}+x_{3}{ }^{2}, x_{1}>0\right\}$. Since $\Phi^{-1}\left(x_{1}, x_{2}, x_{3}\right)=\left(\left(1, \frac{x_{2}}{x_{1}}, \frac{x_{3}}{x_{1}}\right), x_{1}\right), \Phi$ is a diffeomorphism. By the above arguments we may assume that $\mathcal{D}_{\tilde{H}} \subset S_{+}^{1} \times \mathbb{R}_{+}$. It is clear that

$$
\Phi\left(\mathcal{D}_{\tilde{H}}\right)=\{(u \cdot v) \mid v=\langle\gamma, N(s) \widetilde{ \pm} B(s)\rangle \cdot(N(s) \widetilde{ \pm} B(s)), s \in I\} .
$$

By Lemma 4.1 and Theorem $4.2(2)$, the discriminant set $\mathcal{D}_{\tilde{H}}$ of $\widetilde{H}$ is locally diffeomorphic to a line or the cusp. It follows from Proposition 2.1 that the proof of Theorem B (1) (b) is completed.

The assertions (2) (a) is trivial by the definition of the lightcone developable.

For the proof of the assertion (2) (b), (c), we consider the discriminant set of $G$. By Proposition 2.2, the discriminant set of $G$ is given by

$\mathcal{D}_{G}=\left\{v \in \mathbb{R}_{1}^{3} \mid\right.$ there exists $s$ and non-zero real number $\lambda$ with $\left.v=\gamma(s)-\lambda(\boldsymbol{n}(s) \pm \boldsymbol{b}(s))\right\}$.

Hence the assertions follow from Proposition 2.2 and Theorem 4.2.

5. Generic properties of spacelike curves. In this section we consider the notion of Lorentzian Monge-Taylor maps for spacelike curves analogous to the ordinary notion of Monge-Taylor maps for space curves in Euclidean space (cf. [4]).

Firstly we try to choose a pseudo orthonormal frame along $\gamma$ like the Euclidean case. In this case the tangent vector of $\gamma$ is always spacelike, so that the normal plane contains lightlike vectors. We cannot choose a lightlike normal vector as a member of the basis of the frame along $\gamma$. If we construct such a frame, all arguments in the sequel are parallel to those of the Euclidean case. Let $\gamma: I \longrightarrow \mathbb{R}_{1}^{3}$ be a (regular) spacelike curve, with $I$ an open connected subset of the unit circle $S^{1}$, increasing $t$ corresponding to the anticlockwise orientation of $S^{1}$. We now choose a smooth family of unit vectors $\boldsymbol{n}(t)$, with $\boldsymbol{n}(t)$ pseudo-normal to $\gamma$ at $t$, so that $\|\boldsymbol{n}(t)\|=1$ and $\langle\boldsymbol{n}(t), \boldsymbol{t}(t)\rangle=0$, for all $t \in I$. Such $\boldsymbol{n}(t)$ can be obtained as follows: consider the smooth map $\boldsymbol{t}: I \rightarrow S_{1}^{2}$ which takes $t$ to the unit tangent vector $\boldsymbol{t}(t)$. If $V$ is any vector in $H_{1}^{2}$ (where $H_{1}^{2}=\left\{p \in \mathbb{R}_{1}^{3} \mid\langle p, p\rangle=-1\right\}$ ) we can obtain the vector field $\boldsymbol{n}(t)$ by pseudo-orthogonally projecting $V$ onto each of the normal planes and normalizing. Thus $\boldsymbol{n}(t)=\frac{V-\langle V, \boldsymbol{t}(t)\rangle \boldsymbol{t}(t)}{\|V-\langle V, \boldsymbol{t}(t)\rangle \boldsymbol{t}(t)\|}$; then we have $\langle\boldsymbol{n}, \boldsymbol{n}\rangle=-1$ and $\langle\boldsymbol{n}, \boldsymbol{t}\rangle=0$. This means that 
we can choose $\boldsymbol{n}(t)$ as a timelike vector field along $\gamma$. We can obtain a second smooth family of unit vectors $\boldsymbol{b}(t)=\boldsymbol{t}(t) \wedge \boldsymbol{n}(t)$ normal to $\gamma$ at $t$. We remark that the triple $\boldsymbol{t}(t), \boldsymbol{n}(t), \boldsymbol{b}(t)$ is the Lorentzian frame along $\gamma$ and $\boldsymbol{b}(t)$ is spacelike. We now use the pseudo-perpendicular lines spanned by $\boldsymbol{t}(t), \boldsymbol{n}(t), \boldsymbol{b}(t)$ as axes at $\gamma(t)$ with the unit points on the axes corresponding to the three given vectors. We remark that the Minkowski metric is invariant under the Lorentz transformation. Note that the curve $\gamma(t)$ is not necessarily of unit speed, with $\gamma\left(t_{0}\right)=0$. Then the coordinates $\eta, \zeta$ and $\chi$ of $\gamma(t)$ relative to axes $\boldsymbol{t}, \boldsymbol{n}$ and $\boldsymbol{b}$ are functions of $t: \zeta(t)=\gamma(t) \cdot \boldsymbol{t}\left(t_{0}\right), \eta(t)=\gamma(t) \cdot \boldsymbol{n}\left(t_{0}\right)$, $\chi(t)=\gamma(t) \cdot \boldsymbol{b}\left(t_{0}\right), \quad \eta(t)=f(\zeta(t)), \quad \chi(t)=g\left((\zeta(t))\right.$ where $f=f_{0}, g=g_{0}$. So $j^{k} f_{t}(0)=$ $a_{2}(t) \zeta^{2}+a_{3}(t) \zeta^{3}+\cdots+a_{k}(t) \zeta^{k}, \quad j^{k} g_{t}(0)=b_{2}(t) \zeta^{2}+b_{3}(t) \zeta^{3}+\cdots+b_{k}(t) \zeta^{k} \quad$ in the neighbourhood $(0,0,0)$. Locally then $\gamma(I)$ can be written in the form $\left\{\left(f_{t}(\zeta), \zeta, g_{t}(\zeta)\right)\right\}$, with $f(0)=g(0)=j^{1} f_{t}(0)=j^{1} g_{t}(0)=0$. If $V_{k}$ denotes the space of polynomials in $\zeta$ of degree $\geq 2$ and $\leq k$, we have the Lorentzian Monge-Taylor map for the spacelike curve $\gamma, \mu_{\gamma}: I \longrightarrow V_{k} \times V_{k}$ given by $\mu_{\gamma}(t)=\left(j^{k} f_{t}(0), j^{k} g_{t}(0)\right) .\left(V_{k} \times V_{k}\right.$ can be identified with $\mathbb{R}^{k-1} \times \mathbb{R}^{k-1}=\mathbb{R}^{2(k-1)}$ via the coordinates $\left(a_{2}, \ldots, a_{k}, b_{2}, \ldots, b_{k}\right)$.) Of course $\mu_{\gamma}$ depends rather heavily on our choice of unit normals $\boldsymbol{n}(t)$, where $a_{i}(t)=\frac{f_{t}(0)^{(i)}}{i !}$, $b_{i}(t)=\frac{g_{t}(0)^{(i)}}{i !}(2 \leqq i \leqq k)$; that is

$$
V_{k} \times V_{k}=\left\{\left(a_{2} \zeta^{2}+b_{3} \zeta^{3}+\cdots+b_{k} \zeta^{k}\right),\left(b_{2} \zeta^{2}+b_{3} \zeta^{3}+\cdots+a_{k} \zeta^{k}\right)\right\}
$$

Let $P_{k}$ denote the set of maps $\psi: \mathbb{R}_{1}^{3} \rightarrow \mathbb{R}_{1}^{3}$ of the form

$$
\left(\psi(x, y, z)=\left(\psi_{1}(x, y, z), \psi_{2}(x, y, z), \psi_{3}(x, y, z)\right)\right.
$$

where $\psi_{i}(x, y, z)$ is a polynomial in $x, y$ and $z$ of degree $\leqq k$. An element $\psi \in P_{k}$ is determined by the coefficients of the various monomials $x^{l} y^{i} z^{j}$ occurring in $\psi_{1}, \psi_{2}$ and $\psi_{3}$. There are altogether $1+3+\cdots+(2 k-1)+2 k+1=(k+1)^{2}$ monomials of degree $\leqq k$, so that $P_{k}$ can be thought of as a Euclidean space $\mathbb{R}^{(k+1)^{2}}$. It is this space which will provide the required deformations of the curve.

To simplify matters we now assume that the curve $\gamma(I)$ is compact; that is $I=S^{1}$. The identity map $1_{\mathbb{R}_{1}^{3}}: \mathbb{R}_{1}^{3} \rightarrow \mathbb{R}_{1}^{3}$, is of course an element of $P_{k}$ (provided $k \geq 1$ ), and using the compactness of $\gamma\left(S^{1}\right)$ it easily follows that there is a open neighbourhood $U$ of $1_{\mathbb{R}_{1}^{3}}$ in $P_{k}$ with the property that if $\psi \in U$ then the linear map $T \psi(\gamma(t)): \mathbb{R}_{1}^{3} \rightarrow \mathbb{R}_{1}^{3} ; \quad v \mapsto \rightarrow D \psi(\gamma(t)) \cdot v$ is such that $D \psi(\gamma(t)) \cdot \boldsymbol{n}(t)$ is a timelike vector and $D \psi(\gamma(t)) \cdot \boldsymbol{t}(t)$ is a spacelike vector, where $D \psi(\gamma(t))$ denotes the derivative of $\psi$ at $\gamma(t)$. If we deform the original curve by the map $\psi$, then we can also obtain the required new smooth family of normal vectors $\boldsymbol{n}_{\psi}(t)$ as follows. Since the map $\psi: \mathbb{R}_{1}^{3} \rightarrow \mathbb{R}_{1}^{3}$ is a diffeomorphism on some open set containing $\gamma(I)$, the vector $\boldsymbol{n}(t)$ will be sent to some new timelike vector $D \psi(\gamma(t)) n(t)$ which will be neither zero nor tangent to $\psi \circ \gamma$ at $t$. Pseudo-orthogonally project this vector onto the psudo-normal plane to $\psi \circ \gamma$ at $t$ and normalize, that is

$$
\boldsymbol{n}_{\psi}(t)=\frac{D \psi(\gamma(t)) \boldsymbol{n}(t)-\left\langle D \psi(\gamma(t)) \boldsymbol{n}(t), \boldsymbol{t}_{\psi}\right\rangle \boldsymbol{t}_{\psi}}{\left\|D \psi(\gamma(t)) \boldsymbol{n}(t)-\left\langle D \psi(\gamma(t)) \boldsymbol{n}(t), \boldsymbol{t}_{\psi}\right\rangle \boldsymbol{t}_{\psi}\right\|},\left\langle\boldsymbol{n}_{\psi}(t), \boldsymbol{n}_{\psi}(t)\right\rangle=-1,
$$

where $\boldsymbol{t}_{\psi}$ denotes the tangent vector of the curve $\psi \circ \gamma$ at $t$. Assuming as before that $I=S^{1}$, we choose an open neighbourhood $U$ of $1_{S^{1}} \in P_{k}$ consisting of polynomial 
maps which map an open set containing $\gamma\left(S^{1}\right)$ diffeomorphically to its image. We have now shown that there is a smooth map

$$
\mu: S^{1} \times U \longrightarrow V_{k} \times V_{k}
$$

defined by $\mu(-, \psi)=$ Monge-Taylor map for the curve $\psi \circ \gamma$ using the family of normal vectors $\boldsymbol{n}_{\psi}(t)$. By exactly the same arguments as in the proof of Theorem 9.9 in [4], we have the following theorem.

Theorem 5.1. Let $Q$ be a manifold in $V_{k} \times V_{k}=\mathbb{R}^{2 k-2}$. For some open set $U_{1} \subset U$ containing the identity map, the map $\mu: S^{1} \times U_{1} \rightarrow V_{k}$, defined by $\mu(t, \psi)=\mu_{\psi \circ \gamma}(t)$, is transverse to $Q$. (In fact we can prove that $\mu$ is a submersion so that $Q$ does not enter the argument at all.)

By the direct calculations, we have the following lemmas. The calculations are rather long and tedious and so we omit the details.

Lemma 5.2. Let $\gamma: S^{1} \rightarrow \mathbb{R}_{1}^{3}$ be a spacelike curve defined by

$$
\gamma(t)=\left(f_{t}(\zeta), \zeta, g_{t}(\zeta)\right)=\left(a_{2} \zeta^{2}+a_{3} \zeta^{3}+\cdots, \zeta, b_{2} \zeta^{2}+b_{3} \zeta^{3}+\cdots\right)
$$

with $\zeta\left(t_{0}\right)=0$.

(1) $k=0$ and $\left\langle N^{\prime}, N\right\rangle=0$ at $t_{0}$ if and only if $a_{2}{ }^{2}-b_{2}{ }^{2}=0$ and $a_{2} a_{3}-b_{2} b_{3}=0$.

(2) $k^{\prime}\left(s_{0}\right)-k\left(s_{0}\right) \cdot \tau\left(s_{0}\right)=0$ and $\left(k^{\prime}-k \cdot \tau\right)^{\prime}=0$ at $t_{0}$ if and only if

$$
\left\{\begin{array}{l} 
\pm\left(a_{2} a_{3}-b_{2} b_{3}\right)-\left(a_{3} b_{2}-a_{2} b_{3}\right)=0 \\
-3\left(a_{2} a_{3}-b_{2} b_{3}\right)^{2}+\left\{4\left(a_{2} a_{4}-b_{2} b_{4}\right)+3\left(a_{3}^{2}-b_{3}^{2}\right)\right\} \cdot\left|a_{2}^{2}-b_{2}{ }^{2}\right| \\
-4\left(a_{2}^{2}-b_{2}^{2}\right) \cdot\left|a_{2}{ }^{2}-b_{2}^{2}\right|^{2} \mp 3\left(a_{2} a_{3}-b_{2} b_{3}\right) \cdot\left(a_{3} b_{2}-a_{2} b_{3}\right) \\
-4\left|a_{2}{ }^{2}-b_{2}{ }^{2}\right| \cdot\left(a_{4} b_{2}-a_{2} b_{4}\right) \pm 6\left(a_{2} a_{3}-b_{2} b_{3}\right) \cdot\left(a_{3} b_{2}-a_{2} b_{3}\right)=0
\end{array}\right.
$$

where $\zeta$ is the coordinate along the $\boldsymbol{t}$-direction, $f_{t}(\zeta)$ the coordinate along the $\boldsymbol{n}$-direction and $g_{t}(\zeta)$ the coordinate along the b-direction. Moreover, $\left(a_{2}, a_{3}, \cdots, a_{k}, b_{2}, b_{3}\right.$, $\left.\cdots, b_{k}\right) \in \mathbb{R}^{2 k}, k, k^{\prime}$ as in $\S 2$.

LeMma 5.3 We consider smooth maps $\rho_{i}: V_{3} \times V_{3}=\mathbb{R}^{4} \longrightarrow \mathbb{R}(i=1,2)$ given by

$$
\left\{\begin{array}{l}
\rho_{1}=a_{2}^{2}-b_{2}^{2}, \\
\rho_{2}=-b_{2} b_{3}+a_{2} a_{3},
\end{array}\right.
$$

and $\phi_{i}: V_{4} \times V_{4}=\mathbb{R}^{6} \longrightarrow \mathbb{R}(i=1,2)$ given by

$$
\left\{\begin{array}{l}
\phi_{1}= \pm\left(a_{2} a_{3}-b_{2} b_{3}\right)-\left(a_{3} b_{2}-a_{2} b_{3}\right) \\
\phi_{2}=-3\left(a_{2} a_{3}-b_{2} b_{3}\right)^{2}+\left\{4\left(a_{2} a_{4}-b_{2} b_{4}\right)+3\left(a_{3}^{2}-b_{3}^{2}\right)\right\} \cdot\left|a_{2}^{2}-b_{2}^{2}\right|, \\
-4\left(a_{2}^{2}-b_{2}^{2}\right) \cdot\left|a_{2}^{2}-b_{2}^{2}\right|^{2} \mp 3\left(a_{2} a_{3}-b_{2} b_{3}\right) \cdot\left(a_{3} b_{2}-a_{2} b_{3}\right) \\
-4\left|a_{2}^{2}-b_{2}^{2}\right| \cdot\left(a_{4} b_{2}-a_{2} b_{4}\right) \pm 6\left(a_{2} a_{3}-b_{2} b_{3}\right) \cdot\left(a_{3} b_{2}-a_{2} b_{3}\right)
\end{array}\right.
$$


(1) The set $Q_{1}=\left\{\left(a_{2}, a_{3}, b_{2}, b_{3}\right) \in \mathbb{R}^{4} \mid \rho_{1}=\rho_{2}=0\right\}$ is a codimension two submanifold in $\mathbb{R}^{4}$.

(2) The set $Q_{2}=\left\{\left(a_{2}, a_{3}, a_{4}, b_{2}, b_{3}, b_{4}\right) \in \mathbb{R}^{6} \mid \phi_{1}=\phi_{2}=0\right\}$ is a codimension two submanifold in $\mathbb{R}^{6}$.

We can use Theorem 5.1, Lemmas 5.2 and 5.3 for the proof of Theorem A in exactly the same way as the proof of Corollary 9.7 in [4]. We omit the details here.

\section{REFERENCES}

1. J. W. Bruce, On singularities, envelopes and elementary differential geometry, Math. Proc. Cambridge Phil. Soc. 89 (1981), 43-48.

2. J. W. Bruce and P. J. Giblin, Generic curves and surfaces, J. London Math. Soc. 24 (1981), 555-561.

3. J. W. Bruce and P. J. Giblin, Generic geometry, Amer. Math. Monthly 90 (1983), $529-545$.

4. J. W. Bruce and P. J. Giblin, Curves and singularities (second edition) (Cambridge University Press, 1992).

5. D. L. Fidal and P. J. Giblin, Generic 1-parameter families of caustics by reflexion in the plane, Math. Proc. Cambridge Phil. Soc. 96 (1984), 425-432.

6. S. Izumiya and A. Takiyama, A time-like surface in Minkowski 3-space which contains pseudocircles, Proc. Edinburgh Math. Soc. (2) 40 (1997), 127-136.

7. M. Kossowski, The null blow-up of a surface in Minkowski 3-space and intersection in the spacelike Grassman, Michigan Math. J. 38 (1991), 401-415.

8. D. K. H. Mochida, R. C. Romero-Fuster and M. A. Ruas, The geometry of surfaces in 4-space from a contact veiwpoint, Geom. Dedicata 54 (1995), 323-332.

9. B. O’Neill, Semi-Riemannian geometry (Academic Press, New York, 1983). $543-564$

10. I. Porteous, The normal singularities of submanifold, J. Differential Geom. 5 (1971), 